\title{
Triazolopyridines. Part 27. ${ }^{1}$ The preparation of novel 6,7-dihydro[1,2,3]triazolo[1,5-a]pyridines
}

\author{
Belén Abarca, ${ }^{*}$ Rosa Adam, and Rafael Ballesteros* \\ Departamento de Química Orgánica, Facultad de Farmacia, Universidad de Valencia, \\ Avda. Vicente Andrés Estellés s/n, 46100 Burjassot (Valencia), Spain \\ E-mail: belen.abarca@uv.es
}

Dedicated to Professor Benito Alcaide on the occasion of his $60^{\text {th }}$ birthday

DOI: http://dx.doi.org/10.3998/ark.5550190.0011.326

\begin{abstract}
A very efficient synthesis of the unknown family of 6,7-dihydro[1,2,3]triazolo[1,5- $a$ ]pyridines from $[1,2,3]$ triazolo[1,5- $a$ ]pyridines have been developed, and a mechanism for their formation has been proposed. Their behaviour with NBS to give 4,5-dibromo substituted-4,5,6,7tetrahydro-[1,2,3]triazolo[1,5-a]pyridines is studied.
\end{abstract}

Keywords: Nitrogenated heterocycles, triazolopyridines

\section{Introduction}

$[1,2,3]$ Triazolo[1,5-a]pyridines $\mathbf{1}$ are fused biheterocyclic ring systems that are readily accessed by mild oxidation of hydrazones of pyridyl ketones and aldehydes. ${ }^{2}$ Jones and ourselves, have reported on the synthesis of interesting 2,6-disubstituted pyridines, ${ }^{3}$ indolizines, ${ }^{4}$ and bipyridines, ${ }^{5}$ using $[1,2,3]$ triazolo[1,5-a]pyridines as synthons. We have also reported many aspects of the chemistry of $[1,2,3]$ triazolo[1,5-a]pyridines, their fluorescent behaviour, ${ }^{6}$ the preparation of fluorescent sensors of cations and anions, ${ }^{7}$ their coordination chemistry, ${ }^{8}$ and their application in the field of magnetic materials. ${ }^{8 d, 9}$ Recent examples are precursors to pyridyl carbenes, ${ }^{10}$ reagents for transannulation, ${ }^{11}$ or cross-coupling reactions, ${ }^{6,12,13}$ and also chiral fluorescent materials. $^{14}$ 
<smiles>[R]c1nnn2ccccc12</smiles>

1 a $R=H$

b $\mathrm{R}=\mathrm{CH}_{3}$

c $\mathrm{R}=\mathrm{Ph}$

d $R=2-P y$<smiles>[R]c1nnn2c1CCCC2</smiles>

2<smiles>[R]c1nnn2c1C=CCC2</smiles>

3

\section{Figure 1}

Hydrogenation reactions of some [1,2,3]triazolo[1,5-a]pyridines has been studied, under standard conditions $\left(\mathrm{H}_{2}, \mathrm{Pd} / \mathrm{C}, \mathrm{MeOH}\right.$, room temperature $){ }^{15}$ to give 4,5,6,7-tetrahydro$[1,2,3]$ triazolo[1,5-a]pyridines 2 in high yield. In general, the pyridine ring is more easily hydrogenated than the triazole or benzene rings. Also, tetrahydrotriazolopyridines have been obtained in some cases by diazo transfer reactions. ${ }^{16}$

The 6,7-dihydro[1,2,3] triazolo[1,5-a]pyridines $\mathbf{3}$ are unknown compounds, with one exception described in 2004 in the course of a complex reaction, ${ }^{17}$ in which the treatment of 3 phenyl-7-picolinoyl-[1,2,3]triazolo[1,5-a]pyridine 4 with $\mathrm{TsNHNH}_{2}$ in aqueous $\mathrm{NaOH}$ solution at reflux, gave a mixture of the bitriazolopyridine $5(2 \%)$, the alcohol $\mathbf{6}(45 \%)$, the triazolopyridine 1c (15\%), and the surprising 6,7-dihydrotriazolopyridine 3c (38\%) (Scheme 1). We had verified that $\mathbf{3 c}$ is formed from $\mathbf{6}$ in basic medium. ${ }^{17}$

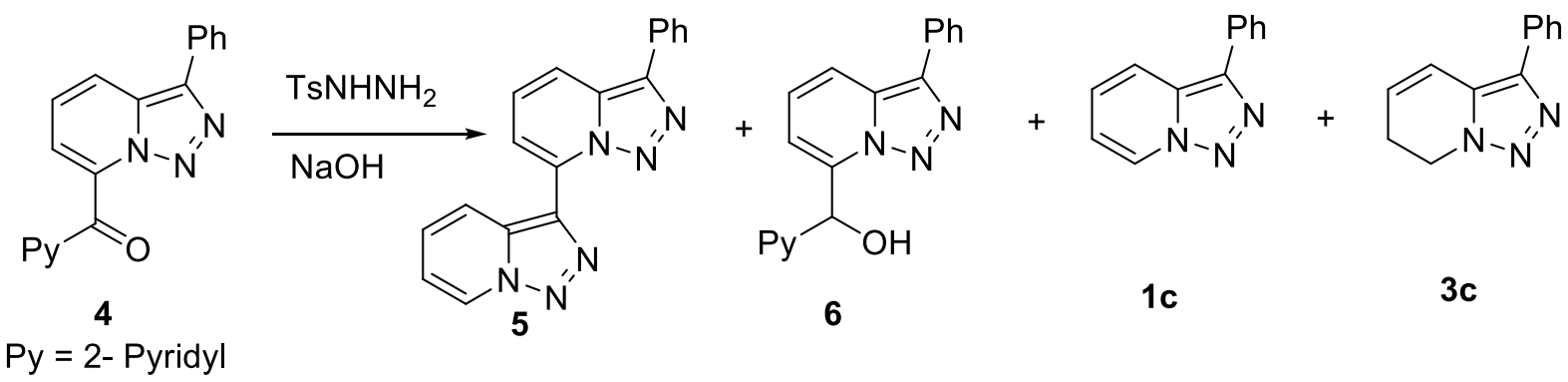

\section{Scheme 1}

We describe in this paper the general synthesis of 6,7-dihydro[1,2,3]triazolo[1,5-a] pyridines 3 from 7-arylhydroxymethyltriazolopyridines 7 in aqueous $\mathrm{NaOH}$ solution at reflux, and we propose a mechanism to explain these results. Also the reaction of 6,7-dihydrotriazolopyridines with NBS is studied. 


\section{Results and Discussion}

To study the scope of the reaction, we have prepared a series of 7-arylhydroxymethyltriazolopyridines 7a-c and we have studied their reactions with $\mathrm{NaOH}$ in ethanol/ water at reflux.

We had reported that the regioselective metalation of $[1,2,3]$ triazolo[1,5-a]pyridines 1a-c with $n$-BuLi in toluene at $-40{ }^{\circ} \mathrm{C}$ gives the corresponding 7-lithioderivatives, which react subsequently with a number of electrophiles to give 7 -substituted triazolo-pyridines. ${ }^{3,17,18}$ Thus, we have synthesized the 7-arylhydroxymethyltriazolopyridines 7 by direct regioselective metalation of triazolopyridines $\mathbf{1}$, followed by treatment with aldehydes. (Scheme 2).
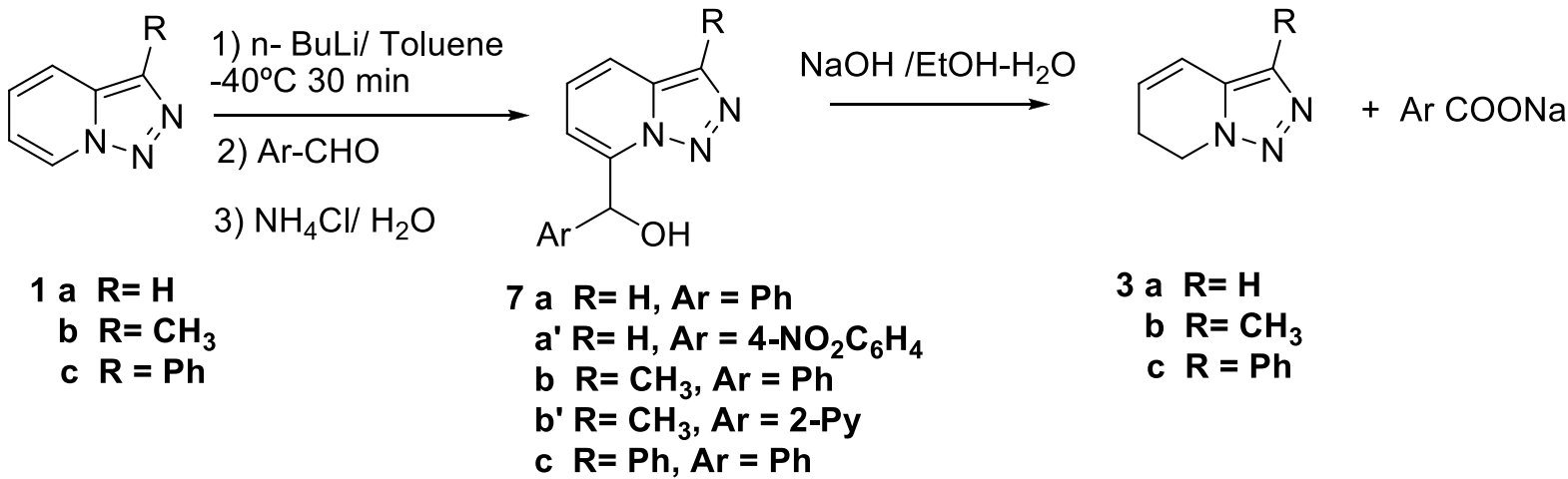

\section{Scheme 2}

With compounds $\mathbf{7 a}, \mathbf{7 b}, \mathbf{7 b}$ ' and $\mathbf{7 c}$ in basic medium we have obtained the 6,7dihydrotriazolopyridines 3a-c in good yield, and sodium benzoate or sodium picolinate as byproducts. In the case of the carbinol 7a' only the triazolopyridine 1a has been obtained in quantitative yield. To explain this behaviour we propose the mechanism shown in Scheme 3 . Deprotonation of alcohols 7 gives the alkoxides 8. Nucleophilic attack to the activated 7-position towards nucleophiles, ${ }^{19}$ and trapping of a proton in C6 position, leads to the intermediates 9 (black arrows). Ring-cleavage reaction with $\mathrm{OH}^{-}$forms the new hemiacetal intermediates 10. By deprotonation in basic media followed by hydride shift to $\mathrm{C} 7$ position, ${ }^{20}$ the 6,7dihydrotriazolopyridines $\mathbf{3}$ are obtained with the corresponding arylcarboxylate salts.

The formation of the triazolopyridine 1a from $7 \mathbf{a}^{\prime}$, could be explained by the formation of alkoxide 8a' and then, a nucleophilic attack of the anion $\left(\mathrm{O}^{-}\right)$to the next $\mathrm{C}$ with formation of a new anion 11 and the corresponding aldehyde (red arrows). Protonation of $\mathbf{1 1}$ gives $\mathbf{1 a}$. 


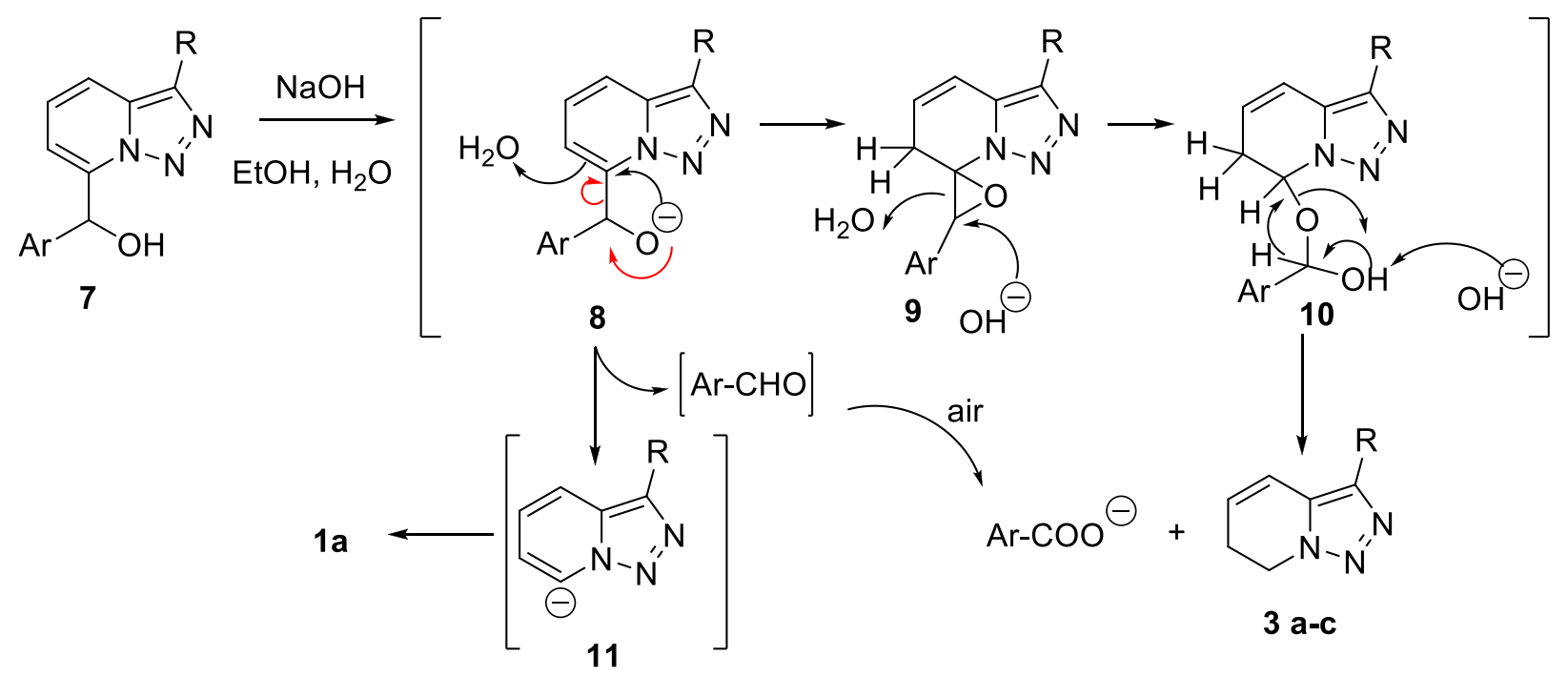

\section{Scheme 3}

Compounds $\mathbf{3 b}$ and $\mathbf{3 c}$ were treated in standard conditions for allylic bromination. ${ }^{21}$ Nevertheless reaction with NBS in carbon tetrachloride and irradiation with visible light gives 12b and 12c in low yields, products of addition of bromine to the double bond (CAUTION carbon tetrachloride, which is an ozone depleting compound and suspected carcinogen, ${ }^{22}$ can be substituted by less pernicious dichloromethane). ${ }^{23,24}$ The corresponding products of allylic bromination were not observed. If two equivalents of NBS and one equivalent of 6,7dihydrotriazolopyridines were used only polymerization was observed. By this procedure it was able to prepare new 4,5-dibromo-4,5,6,7-tetrahydrotriazolopyridines 12b and 12c. (Scheme 4).<smiles>[R]c1nnn2c1C=CCC2</smiles>

3 b,c

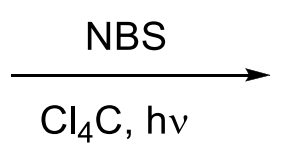

$\mathrm{Cl}_{4} \mathrm{C}, \mathrm{hv}$

(<smiles>[R]c1nnn2c1[C@H](Br)[C@H](Br)CC2</smiles>

12 b $(34 \%)$

\section{Scheme 4}

\section{Conclusions}

In summary we have developed an efficient synthesis of the unknown family of 6,7dihydrotriazolopyridines. We have proposed a mechanism for their formation and we have studied their behaviour with NBS to give 4,5-dibromo substituted tetrahydrotriazolopyridines. 


\section{Experimental Section}

General Procedures. Melting points were determined on a Kofler heated stage and are uncorrected. NMR spectra were recorded on a Bruker $\mathrm{AC} 300 \mathrm{MHz}$ in $\mathrm{CDCl}_{3}$ as solvent. COSY experiments were done for all compounds. HRMS Electron Impact (EI) or ElectroSpray (ES) determinations were made using a VG Autospec Trio 1000 (Fisons). All the lithiation reactions were done under inert atmosphere and dry solvents. ${ }^{25}$

$[1,2,3]$ Triazolo[1,5-a]pyridine $\mathbf{1 a},{ }^{26}$ 3-methyl-[1,2,3]triazolo[1,5-a]pyridine $\mathbf{1 b},{ }^{26}$ 3-phenyl$[1,2,3]$ triazolo[1,5-a]pyridine $\mathbf{1 c},{ }^{26}$ phenyl $[1,2,3]$ triazolo[1,5-a]pyridin-7-yl methanol 7a ${ }^{3}$ 4nitrophenyl [1,2,3]triazolo[1,5-a]pyridin-7-yl methanol 7a', ${ }^{3}$ phenyl 3-methyl[1,2,3]triazolo[1,5-a]pyridin-7-yl methanol 7b, and 2-pyridyl 3-methyl-[1,2,3] triazolo[1,5a]pyridin-7-yl methanol 7b', ${ }^{18}$ were prepared as described elsewhere.

Phenyl 3-phenyl-[1,2,3]triazolo[1,5-a]pyridin-7-yl methanol (7c). To a solution of 3-phenyl$[1,2,3]$ triazolo[1,5-a]pyridine $1 \mathrm{c}(1 \mathrm{~g}, 5.12 \mathrm{mg})$ in anhydrous toluene $(57 \mathrm{ml})$ at $-40^{\circ} \mathrm{C}$, a solution of $n$-butyllitium in hexane $(3.2 \mathrm{ml}, 1.6 \mathrm{M})(2.5 \mathrm{M})$ was added with stirring. A deep red colour developed. The mixture was kept at $-40^{\circ} \mathrm{C}(30 \mathrm{~min})$. Treatment with benzaldehyde $(0.54 \mathrm{~g}, 5.12$ $\mathrm{mmol}$ ) produced a colour change to yellow. The reaction mixture was left at room temperature ( 2 h $30 \mathrm{~min}$ ), and quenched with a saturated aqueous solution of ammonium chloride. The organic layer was separated and the aqueous layer extracted with dichloromethane. After dried over anhydrous $\mathrm{Na}_{2} \mathrm{SO}_{4}$ and evaporation of the organic solvents, a residue was obtained. This residue was purified by FLASH $+40^{\mathrm{TM}}$ chromatography (Biotage cartridges) over silica gel with hexaneethyl acetate to obtain 7c (500 mg, 32.4\%). HMRS found for $\mathrm{M}^{+} 301.1206 ; \mathrm{C}_{19} \mathrm{H}_{15} \mathrm{~N}_{3} \mathrm{O}$ requires 301.1215. ${ }^{1} \mathrm{H}$ NMR $\delta 7.98-7.94(\mathrm{~m}, 3 \mathrm{H}), 7.60\left(\mathrm{dd}, \mathrm{J}_{1}=8.1 \mathrm{~Hz}, \mathrm{~J}_{2}=1.14 \mathrm{~Hz}, 2 \mathrm{H}\right), 7.52\left(\mathrm{t}, \mathrm{J}_{1}=\right.$ $7.23 \mathrm{~Hz}, 2 \mathrm{H}), 7.45-7.29(\mathrm{~m}, 5 \mathrm{H}), 6.77(\mathrm{~d}, \mathrm{~J}=6.87 \mathrm{~Hz}, 1 \mathrm{H}), 6.58(\mathrm{~s}, 1 \mathrm{H}), 4.67(\mathrm{~s}, 1 \mathrm{H}, \underline{\mathrm{OHCH}})$. ${ }^{13} \mathrm{C}$ NMR $\delta 139.13(\mathrm{C}), 137.14(\mathrm{C}), 137.07(\mathrm{C}), 129.92(\mathrm{C}), 129.62(\mathrm{C}), 127.75(\mathrm{CH}), 127.34$ $(\mathrm{CH}), 127.27(\mathrm{CH}), 126.76(\mathrm{CH}), 125.95(\mathrm{CH}), 125.46(\mathrm{CH}), 124.68(\mathrm{CH}), 116.08(\mathrm{CH}), 112.05$ (CH), 70.21 (CH). EM (E.I.): m/z (\%) 301 (5.6); 273 (84.5); 244 (100.0); 228 (8.6); 196 (26.6); 182 (5.0); 168 (70.2); 139 (58.6); 105 (71.7); 91 (12.4) ; 77 (99.2).

\section{General procedure for preparation of 6,7-dihydrotriazolopyridines}

To a solution of the corresponding 7-arylhydroxymethyltriazolopyridine $\mathbf{7} \mathbf{a}, \mathbf{b}, \mathbf{b}$ ' $\mathbf{c}$ in ethanol was added a solution of aqueous $\mathrm{NaOH}$ (conditions in Table 1), and the mixture was heated to reflux $\left(95^{\circ} \mathrm{C}\right)$ during 24 hours. The ethanol was evaporated, and the aqueous layer was extracted with dichloromethane. The organic phase was dried with $\mathrm{Na}_{2} \mathrm{SO}_{4}$ and concentrated, giving a crude mixture. The products, yields, and conditions of purification are given for each compound. 
Table 1. Conditions for the reaction of 7-arylhydroxymethyltriazolopyridines with $\mathrm{NaOH}$

\begin{tabular}{llll}
\hline 7-Arylhydroxymethyl triazolopyridine & $(\mathrm{mg}, \mathrm{mmol})$ & $\mathrm{NaOH}$ aq $(\mathrm{ml}, \mathrm{M})$ & $\mathrm{EtOH}(\mathrm{ml})$ \\
\hline $\mathbf{7 a}$ & $(170,0.75)$ & $(27,4)$ & 25 \\
$\mathbf{7 b}$ & $(350,1.44)$ & $(50,4)$ & 50 \\
$\mathbf{7 b}$, & $(100,0.42)$ & $(15,4)$ & 15 \\
$\mathbf{7 c}$ & $(500,1.66)$ & $(60,4)$ & 50 \\
\hline
\end{tabular}

6,7-Dihydro[1,2,3]triazolo[1,5-a]pyridine (3a). The crude product from 7a was purified by chromatotron with ethyl acetate/hexane (51.06 mg, 56.3\%, colourless oil). HMRS found for $\mathrm{M}^{+}$ 121.0642; $\mathrm{C}_{6} \mathrm{H}_{7} \mathrm{~N}_{3}$ requires 121.0640. ${ }^{1} \mathrm{H}$ NMR $\delta 7.35(\mathrm{~s}, \mathrm{H} 3), 6.53\left(\mathrm{ddd}, \mathrm{J}_{1}=9.8 \mathrm{~Hz}, \mathrm{~J}_{2}=\mathrm{J}_{3}=1.8 \mathrm{~Hz}\right.$, $1 \mathrm{H}, \mathrm{H} 4), 6.11$ (ddd, $\left.\mathrm{J}_{1}=9.61 \mathrm{~Hz}, \mathrm{~J}_{2}=4.3 \mathrm{~Hz}, \mathrm{~J}_{3}=4.5 \mathrm{~Hz}, 1 \mathrm{H}, \mathrm{H} 5\right), 4.48$ (t, $\left.\mathrm{J}_{1}=7.8 \mathrm{~Hz}, 2 \mathrm{H}, \mathrm{H} 7\right), 2.70$ (m, 2H, H6). ${ }^{13} \mathrm{C}$ NMR $\delta 132.11(\mathrm{C}), 129.48(\mathrm{CH}), 127.17(\mathrm{CH}), 115.48(\mathrm{CH}), 43.65\left(\mathrm{CH}_{2}\right)$, $24.52\left(\mathrm{CH}_{2}\right)$. MS (E.I.): m/z (\%) 121 (100), 93 (23), 92 (24), 67 (17), 66 (72), 65 (51), 53 (9).

3-Methyl-6,7-dihydro[1,2,3]triazolo[1,5-a]pyridine (3b). The crude product from $\mathbf{7 b}$ was purified by chromatotron with ethyl acetate/hexane $(94.6 \mathrm{mg}, 50 \%$, colourless oil), and from $\mathbf{7 b}$ ' was purified in the same conditions (20 mg, 48\%).). HMRS (ES) found for $\left(\mathrm{M}^{+}+1\right)$ 136.0873; $\mathrm{C}_{7} \mathrm{H}_{9} \mathrm{~N}_{3} \mathrm{H}$ requires 136.0875. ${ }^{1} \mathrm{H}$ NMR $\delta 6.36$ (ddd, $\left.\mathrm{J}_{1}=9.9, \mathrm{~J}_{2}=\mathrm{J}_{3}=1.8 \mathrm{~Hz}, 1 \mathrm{H}, \mathrm{H} 4\right), 5.96$ (ddd, $\left.\mathrm{J}_{1}=9.9, \mathrm{~J}_{2}=\mathrm{J}_{3}=4.5 \mathrm{~Hz}, 1 \mathrm{H}, \mathrm{H} 5\right), 4.32\left(\mathrm{t}, \mathrm{J}_{1}=\mathrm{J}_{2}=7.8 \mathrm{~Hz}, 2 \mathrm{H}, \mathrm{H} 7\right), 2.57(\mathrm{~m}, 2 \mathrm{H}, \mathrm{H} 6), 2.23(\mathrm{~s}, 3 \mathrm{H}$, $\left.\mathrm{CH}_{3}\right) .{ }^{13} \mathrm{C}$ NMR $\delta 132.20(\mathrm{C}), 128.80(\mathrm{C}), 125.59(\mathrm{CH}), 115.08(\mathrm{CH}), 43.86\left(\mathrm{CH}_{2}\right), 23.90\left(\mathrm{CH}_{2}\right)$, $9.84\left(\mathrm{CH}_{3}\right)$. MS (E.I.): m/z (\%) 135 (100), 107 (70), 106 (81), 92 (19), 79 (90), 66 (83).

3-Phenyl-6,7-dihydro[1,2,3]triazolo[1,5-a]pyridine (3c). The crude product from 7c was purified by chromatotron with ethyl acetate/hexane (90.4 mg, 28\%, yellow solid). Mp 100-102 ${ }^{\circ} \mathrm{C}$ (hexane). HMRS (ES) found for $\left(\mathrm{M}^{+}+1\right)$ 198.1029; $\mathrm{C}_{12} \mathrm{H}_{11} \mathrm{~N}_{3} \mathrm{H}$ requires 198.1031. ${ }^{1} \mathrm{H}$ NMR $\delta 7.75\left(\mathrm{dd}, \mathrm{J}_{1}=7.14 \mathrm{~Hz}, \mathrm{~J}_{2}=1.5 \mathrm{~Hz}, 2 \mathrm{H}, \mathrm{Ho}\right), 7.45\left(\mathrm{dd}, \mathrm{J}_{1}=\mathrm{J}_{2}=7.14 \mathrm{~Hz}, 2 \mathrm{H}, \mathrm{Hm}\right), 7.36(\mathrm{dd}$, $\left.\mathrm{J}_{1}=7.14 \mathrm{~Hz}, \mathrm{~J}_{2}=1.5 \mathrm{~Hz}, 1 \mathrm{H}, \mathrm{H} p\right), 6.78\left(\mathrm{ddd}, \mathrm{J}_{1}=9.99 \mathrm{~Hz}, \mathrm{~J}_{2}=\mathrm{J}_{3}=1.89 \mathrm{~Hz}, 1 \mathrm{H}, \mathrm{H} 4\right), 6.19$ (ddd, $\left.\mathrm{J}_{1}=9.99 \mathrm{~Hz}, \mathrm{~J}_{2}=\mathrm{J}_{3}=4.35 \mathrm{~Hz}, 1 \mathrm{H}, \mathrm{H} 5\right), 4.45\left(\mathrm{t}, \mathrm{J}=7.74 \mathrm{~Hz}, 2 \mathrm{H}, \mathrm{H} 7, \mathrm{H} 7\right.$ '), 2.67 (m, 2H, H6, H6'). ${ }^{13} \mathrm{C}$ NMR $\delta 142.06(\mathrm{C}), 131.23(\mathrm{C}), 128.85(2 \mathrm{CH}), 127.97(\mathrm{CH}), 127.58(\mathrm{CH}), 126.93(2 \mathrm{CH}), 126.17$ (C), $116.25(\mathrm{CH}), 44.09\left(\mathrm{CH}_{2}\right), 23.92\left(\mathrm{CH}_{2}\right)$.

\section{General procedure for preparation of 4,5-dibromo-4,5,6,7-tetrahydrotriazolopyridines}

To a solution of the corresponding 6,7-dihydro-[1,2,3]triazolo[1,5-a]pyridine $\mathbf{3 b}$, $\mathbf{c}$ in $\mathrm{CCl}_{4}$ $(10 \mathrm{ml})$ was added NBS (1 eq). The mixture was heated to reflux and irradiated with visible light (conditions showed in table 2). Formation of succinimide was observed, which floated on the surface of the $\mathrm{CCl}_{4}$. The solution was cooled to room temperature and the succinimide was removed by filtration and washed with $\mathrm{CCl}_{4}$. The combined filtrates were concentrated under reduced pressure giving a crude mixture. The products, yields and conditions of purification are given for each compound. 
Table 2. Conditions for the reaction of 6,7-dihydro[1,2,3]triazolo[1,5- $a]$ pyridine with NBS

\begin{tabular}{lll}
\hline 6,7-Dihydro-[1,2,3]triazolo[1,5-a]pyridine (mg, mmol) & NBS $(\mathrm{mg}, \mathrm{mmol})$ & time \\
\hline 3b $(112,0.83)$ & $(144,0.83)$ & $5 \mathrm{~h}$ \\
3c $(90.4,0.46)$ & $(79.8,0.46)$ & $6 \mathrm{~h}$
\end{tabular}

4,5-Dibromo-3-methyl-4,5,6,7-tetrahydro[1,2,3]triazolo[1,5-a]pyridine (12b). The crude product was purified by chromatotron with ethyl acetate/hexane (73.6 mg, 34\%, yellow oil). HMRS found for $\mathrm{M}^{+} 292.9162 ; \mathrm{C}_{7} \mathrm{H}_{9} \mathrm{Br}_{2} \mathrm{~N}_{3}$ requires 292.9163. ${ }^{1} \mathrm{H}$ NMR $\delta 5.54(\mathrm{~m}, 1 \mathrm{H}, \mathrm{H} 4), 4.85$ $(\mathrm{m}, 1 \mathrm{H}, \mathrm{H} 5), 4.73\left(\mathrm{ddd}, \mathrm{J}_{1}=13.7 \mathrm{~Hz}, \mathrm{~J}_{2}=6.2 \mathrm{~Hz}, \mathrm{~J}_{3}=1.4 \mathrm{~Hz}, 1 \mathrm{H}, \mathrm{H} 7\right), 4.57$ (ddd, $\mathrm{J}_{1}=13.6 \mathrm{~Hz}$, $\left.\mathrm{J}_{2}=12.2 \mathrm{~Hz}, \mathrm{~J}_{3}=4.7 \mathrm{~Hz}, 1 \mathrm{H}, \mathrm{H} 7\right), 2.99(\mathrm{~m}, 1 \mathrm{H}, \mathrm{H} 6), 2.40(\mathrm{~m}, 1 \mathrm{H}, \mathrm{H} 6), 2.30\left(\mathrm{~s}, 3 \mathrm{H}, \mathrm{CH}_{3}\right) .{ }^{13} \mathrm{C} \mathrm{NMR}$ $142.68(\mathrm{C}), 127.61(\mathrm{C}), 46.29(\mathrm{CH}), 42.52\left(\mathrm{CH}_{2}\right), 39.18(\mathrm{CH}), 25.58\left(\mathrm{CH}_{2}\right), 10.28\left(\mathrm{CH}_{3}\right) . \mathrm{MS}$ (E.I.): m/z (\%) 292 (3.5); 214 (63.8); 186 (23.5); 135 (100); 107 (88.4); 79 (31.5); 168 (70.2); 139 (58.6); 105 (71.7); 91 (12.4); 77 (99.2).

4,5-Dibromo-3-phenyl-4,5,6,7-tetrahydro[1,2,3]triazolo[1,5-a]pyridine (12c). The crude product was purified by chromatotron with ethyl acetate/hexane (43.4 mg, 27\%, yellow solid). Mp 138-142 ${ }^{\circ} \mathrm{C}$ (AcOEt/hexane). HMRS found for $\mathrm{M}^{+}$354.9314; $\mathrm{C}_{12} \mathrm{H}_{11} \mathrm{Br}_{2} \mathrm{~N}_{3}$ requires 354.9320. ${ }^{1} \mathrm{H}$ NMR $\delta 7.92\left(\mathrm{dd}, \mathrm{J}_{1}=8.2 \mathrm{~Hz}, \mathrm{~J}_{2}=1.5 \mathrm{~Hz}, 2 \mathrm{H}, \mathrm{H} 1{ }^{\prime}, \mathrm{H} 5{ }^{\prime}\right), 7.50$ (ddd, $\mathrm{J}_{1}=7.6, \mathrm{~J}_{2}=6.1 \mathrm{~Hz}$, $\mathrm{J}_{3}=1.4 \mathrm{~Hz}, 2 \mathrm{H}, \mathrm{H} 2$ ', H4'), 7.41 (ddd, $\mathrm{J}_{1}=\mathrm{J}_{2}=6.4 \mathrm{~Hz}, \mathrm{~J}_{3}=1.3 \mathrm{~Hz}, 1 \mathrm{H}, \mathrm{H} 3$ '), 5.79 (m, 1H, H4), 4.93 $(\mathrm{m}, 1 \mathrm{H}, \mathrm{H} 5), 4.87\left(\mathrm{ddd}, \mathrm{J}_{1}=13.7 \mathrm{~Hz}, \mathrm{~J}_{2}=6.6 \mathrm{~Hz}, \mathrm{~J}_{3}=1.2 \mathrm{~Hz}, 1 \mathrm{H}, \mathrm{H} 7\right), 4.69$ (ddd, $\mathrm{J}_{1}=13.7 \mathrm{~Hz}$, $\left.\mathrm{J}_{2}=12.4 \mathrm{~Hz}, \mathrm{~J}_{3}=5.2 \mathrm{~Hz}, 1 \mathrm{H}, \mathrm{H} 7\right), 3.20(\mathrm{~m}, 1 \mathrm{H}, \mathrm{H6}), 2.46(\mathrm{~m}, \mathrm{~J}=14 \mathrm{~Hz}, 1 \mathrm{H}, \mathrm{H} 6) .{ }^{13} \mathrm{C} \mathrm{NMR} \delta 145.28$ (C), $137.45(\mathrm{C}), 130.58(\mathrm{C}), 129.36(\mathrm{CH}), 128.91(\mathrm{CH}), 127.45(\mathrm{CH}), 46.41(\mathrm{CH}), 43.29\left(\mathrm{CH}_{2}\right)$, $41.17(\mathrm{CH}), 25.70\left(\mathrm{CH}_{2}\right)$. MS (E.I.): m/z (\%) 197 (9.5); 276 (3.3); 169 (100); 154 (17.0); 141 (20.4); 128 (9.2); 115 (27.2); 104 (11.8); 102 (4.9).

\section{Acknowledgements}

We are grateful to the Ministerio de Educación y Ciencia (Spain) (Project CTQ2006-15672-C0503) for its financial support, and to the SCSIE for the realization of the HRMS spectra. R. A. thanks Generalitat Valenciana for a predoctoral fellowship.

\section{References}

1. Abarca, B.; Ballesteros, R.; Ballesteros-Garrido, R.; Colobert, F.; Leroux, F. R. Tetrahedron 2008, 64, 3794.

2. (a) Jones, G.; Sliskovic, D. R. Adv. Heterocycl. Chem. 1983, 34, 79. (b) Jones, G. Adv. Heterocycl. Chem. 2002, 83, 1. (c) Abarca, B. J. Enz. Inh. Med. Chem. 2002, 17, 359.

3. Jones, G.; Sliskovic, R. J. Chem. Soc. Perkin I 1982, 967.

4. Jones, G.; Abarca, B.; Ballesteros, R. Heterocycles 1992, 33, 203. 
5. (a) Jones, G.; Pitman, M. A.; Lunt, E.; Lyghgoe, D. J.; Abarca B.; Ballesteros R.; Elmasnouy, M. Tetrahedron 1997, 53, 8257. (b) Abarca, B.; Ballesteros, R.; Elmasnaouy, M. Tetrahedron 1998, 54, 15287.

6. Abarca, B.; Aucejo, R.; Ballesteros, R.; Blanco, F.; García-España, E. Tetrahedron Lett. 2006, 47, 8101 .

7. (a) Chadlaoui, M.; Abarca, B.; Ballesteros, R.; Ramírez de Arellano, C.; Aguilar, J.; Aucejo, R.; García-España, E. J. Org. Chem. 2006, 71, 9030. (b) Ballesteros-Garrido, R.; Abarca, B.; Ballesteros, R.; Ramirez de Arellano, C.; Leroux, F. R.; Colobert, F.; García-España, E. New J. Chem. 2009, In press. DOI 10.1039/b906992e.

8. (a) Battaglia, L. P.; Carcelli, M.; Ferraro, F.; Mavilla, L.; Pellizzi, C.; Pellizzi, G. J. Chem. Soc. Dalton Trans. 1994, 2651. (b) Ballesteros, R.; Abarca, B.; Samadi, A.; Server-Carrió, J.; Escrivá, E. Polyhedron 1999, 18, 3129. (c) Niel, V.; Gaspar, A. B.; Muñoz, M. C.; Abarca, B.; Ballesteros, R.; Real, J. A. Inor. Chem. 2003, 42, 4782. (d) Abarca, B.; Ballesteros, R.; Chadlaoui, M.; Ramírez de Arellano, C.; Real, J. A. Eur. J. Inorg. Chem. 2007, 4574. (e) Fitchett, C. M.; Keene, F. R.; Richardson, C.; Steel, P. J. Inorg. Chem. Commun. 2008, 11, 595.

9. (a) Boudalis, A. K.; Raptopoulou, C. P.; Abarca, B.; Ballesteros, R.; Chadlaoui, M.; Tuchages, J-P.; Terzis, A. Angew. Chem. Int. Ed. Engl. 2006, 45, 432. (b) Boudalis, A. K.; Raptopoulou, C. P.; Psycharis, V.; Sanakis, Y.; Abarca, B.; Ballesteros, R.; Chadlaoui, M. Dalton Trans. 2007, 3582. (c) Boudalis, A. K.; Raptopoulou, C. P.; Psycharis, V.; Abarca, B.; Ballesteros, R. Eur. J. Inorg. Chem. 2008, 3796. (d) Boudalis, A. K.; Pissas, M.; Raptopoulou, C.P.; Psycharis, V. Abarca, B.; Ballesteros, R. Inorg. Chem. 2008, 47, 10674.

10. Abarca, B.; Ballesteros, R.; Blanco, F. Arkivoc 2007, (iv), 297.

11. Chuprakov, S.; Hwang, F. W.; Gevorgyan, V. Angew. Chem. Int. Ed. 2007, 46, 4757.

12. Abarca, B.; Ballesteros, R.; Blanco, F.; Bouillon, A.; Collot, V.; Dominguez, J. R.; Lancelot, J. C.; Rault. S. Tetrahedron 2004, 60, 4887.

13. (a) Ballesteros-Garrido, R.; Abarca, B.; Ballesteros, R.; Leroux, F. R.; Colobert, F. Tetrahedron Lett. 2007, 48, 6896. (b) Ballesteros-Garrido, R.; Abarca, B.; Ballesteros, R.; Colobert, F.; Leroux, F. R. Tetrahedron 2008, 64, 3794.

14. Ballesteros-Garrido, R.; Abarca, B.; Ballesteros, R.; Colobert, F.; Leroux, F. R. Tetrahedron 2007, 63, 10479.

15. Abarca, B.; Ballesteros, R.; Elmasnouy, M. Tetrahedron 1999, 55, 12881.

16. Augusti, R.; Kascheres, C. Tetrahedron 1994, 50, 6723.

17. Abarca, B.; Ballesteros, R.; Chadlaoui, M. Tetrahedron 2004, 60, 5785.

18. Abarca, B.; Ballesteros, R.; Elmasnouy, M. Arkivoc 2002, (x), 9.

19. Nucleophilic substitution in triazolopyridines has been described, see (a) Abarca, B.; Ballesteros, R.; Jones, G.; Mojarrad, F. Tetrahedron Lett. 1986, 27, 3543. (b) Abarca, B.; Ballesteros, R.; Jones, G.; Mojarrad, F. Tetrahedron 1988, 44, 3005. (c) Reference 5. 
20. Recently we have described a similar rearrangement in $\mathrm{C} 7$ position. Abarca, B.; Aucejo, R.; Ballesteros, R.; Chadlaoui, M.; García-España, E.; Ramírez de Arellano, C. Arkivoc 2005, (xiv), 71.

21. Paquette, L. A. Encyclopedia of Reagents for Organic Synthesis. John Wiley \& Sons: Chichester, 1995; Vol 1, p 768.

22. We are grateful to one of the referees for his/her comments about the pernicious properties of carbon tetrachloride.

23. Yamada, M.; Ichikawa, T.; Ii, M.; Itoh, K.; Tamura, N.; Kitazaki, T. Biorg. Med. Chem. 2008, 16, 3941.

24. Meyers, D. J.; Fucha, P. L. J. Org. Chem. 2002, 67, 200.

25. Perrin, D.; Armarego, L. F. Purification of Laboratory Chemicals, 3rd Ed.; Pergamon: Oxford, 1988.

26. Bower, D. J.; Ramage, G. R. J. Chem. Soc. 1957, 4506. Note that they are now commercially available (ASM Research Chemicals). 